\title{
Sports motion analysis based on mobile sensing technology
}

\author{
YU Wei, QINGSONG Fei, LIJUAN He \\ Computer Teaching Section, Capital Institute of Physical Education, Beijing, China \\ \{weiyu, feiqingsong, helijuan \}@cupes.edu.cn
}

\begin{abstract}
Motion analysis plays a significant role in most of the sports, a large number of demands of Information Technology exist in related fields, including the sports training and P.E. practicing. As the development of mobile smart devices and relative software applications, we are able to assist sports practicing and training more easily and freely. Here, in this paper, we are going to focus on the application of sensor within smart phone in sports motion evaluation, such as the estimation of gymnastics gestures, and we are going to propose a solution scheme on sports motion evaluation.
\end{abstract}

\section{Introduction}

Information Technology has long be applied in sports teaching and training process, for example, camera technology as well as the 3D techniques are used in visualization of body motion of trainers. Nowadays, wireless sensors are commonly used in many fields, including sports area. For example, wearable Body Area Network can be used to capture human motions as well as the body state. However, many of these existing assistant tools are either cost too much or inconvenience for ordinary practice. Since smart phone is quite popular in daily life, mobile application plays an important role in many aspects. For example, there are many mobile health and fitness applications that help people to do body building in an easy and flexible way. Its portability and intelligence show the advantage of related techniques.

Motion evaluation is needed in most of the sports items, which is usually done by coaches or teachers visually at present. However, it is not possible for the experts to be with trainers all the time during the training process. Furthermore, teachers are unable to watch every student's practice after class, while their homework needs to be checked. Besides, there are also requirements from normal exercisers without any mentors to check their performance. Therefore, we decide to apply mobile sensing technology in this field, especially with the help of build-in sensors of smart phone. In fact, there are many related researches involve in motion recognition ${ }^{[1,2,3]}$ which can be used for reference in our study. There are Intelligent evaluation systems designed for team sports ${ }^{[4,5]}$, and most of them are video-based, computer-aided. Here, in this paper, we apply the accelerometer within the smart phone to capture the movement of human body and estimate the correctness of the gestures and rhythm by comparing the test samples with the standard model.

\section{Mobile Sensing Technology}

\footnotetext{
* This work is supported by the Youth Talents Project of Beijing (YETP1711).
}

As the development of Internet of Things, related technologies such as mobile sensing, RFID, and Cloud Computing start to boom. Mobile devices and wireless sensors are widely used in many fields, including sport training and daily exercises. For example, wireless sensors are used in training aiding, such as the BSN based Golf training system ${ }^{[6,7]}$ which can provide feedback for trainer to improve their wrist motion. Since most of the wireless sensors are portable and wearable, these studies possess wider application scope than the traditional ones that rely on visual tools. Compare to the trainings of professional athletes that require high accuracy of the measuring, simple configuration of the hardware which is easy to use with low cost is more practical for practice of normal students and ordinary exercisers. So, smart phone become a wise choice, which has prepared technically ${ }^{[8]}$. In fact, there are a lot of applications involved in mobile health and fitness aiding exist on IOS as well as Android platform ${ }^{[9]}$.

\section{Solution Scheme on motion evaluation}

In order to estimate the correctness of sports motions, we design an evaluation system based on the $3 \mathrm{D}$ accelerometer within the smart phone to assist gymnastics practice. It can capture the continuous human motion and compare with the standard model so as to check the performance of gestures. The architecture of the system is demonstrated as below(Fig.1):

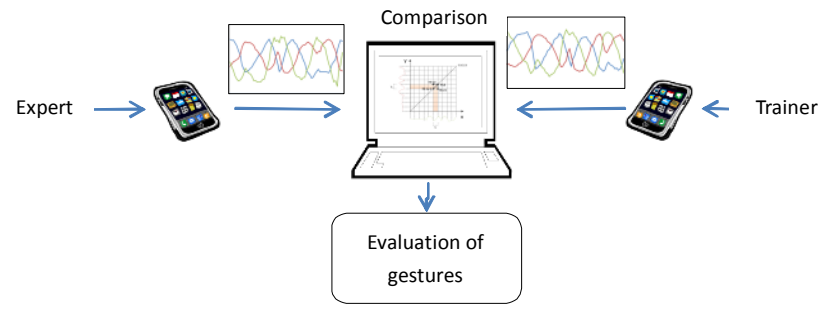

Fig. 1 the architecture of the system

The process of the system is mainly consisted of 4 parts. First of all, correct motions performed by experts will be collected by $3 \mathrm{D}$ accelerometers of smart phones and used to train the standard model. Then, motions of trainers will be obtained by smart phone as well. Thirdly and most importantly, comparison between trainers' motion and standard model is done by the executing the algorithm based on Euclidean Distance 
measurement. The definition of Euclidean Distance between two data segments is shown as follows:

$$
\|x-y\|=\operatorname{sqrt}\left[\sum_{i=1}^{n}\left(x_{i}-y_{i}\right)^{2}\right]
$$

Here, the data segments are indicated as $x$ and $y$, and they are both $n$ dimensional vectors.

Furthermore, in order to check the situation of rhythm management, we apply Dynamic Time Warping to test the synchronism of the signals. This method is easier and more efficient than Hidden Markov Model which is commonly used in detecting synchronism of continuous data signals. After the comparison, the correctness of gestures is measured by their distance to the standard model which can be classified into different grades(shown in Fig.2). Here, the four categories represent four levels: A for Excellent, B for Good, C for Average, and D for Below Average. In Fig.2, 10 samples are grouped into four grades.

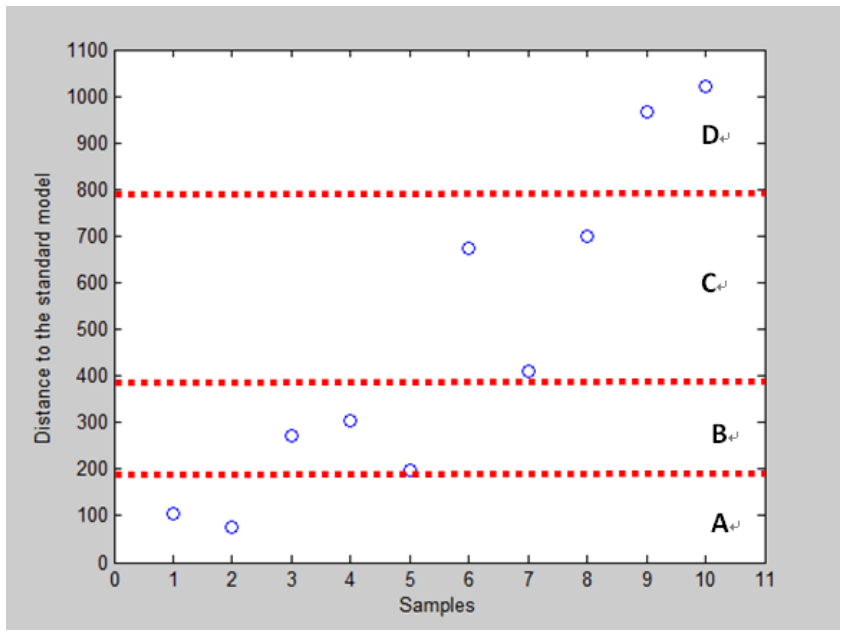

Fig. 2 four levels of gesture performance

This scheme is designed based on the demands of gymnastics training, in which the performance can be measured by the movement of certain parts of the body. For some simple training segments, we may need only one accelerometer to obtain the motion of the most important part of the body, while other complex ones might need to add more sensors to form a sensor network. Whatever it is, this method is easy to be extended for different requirements. Meanwhile, it is quite flexible for it suits various kinds of motions by modifying the threshold of the classification.

\section{Experiment}

In order to investigate the feasibility and related performance of the application, a few experiments are carried out in students gymnastics training with the scheme we designed. First of all, we choose 5 testers to performance a set of actions, and comparison is done between the evaluations from a professional teacher and our system. The results of the comparison are shown in Tab.1.

Tab. 1 the evaluation results compared between expert and computer

\begin{tabular}{|c|c|c|}
\hline \multirow{2}{*}{$\begin{array}{c}\text { Test sample } \\
\text { ID }\end{array}$} & Result from expert & Result from computer \\
\cline { 2 - 3 } & gesture & gesture \\
\hline 1 & A & A \\
\hline 2 & B & B \\
\hline 3 & B & B \\
\hline 4 & C & C \\
\hline 5 & D & D \\
\hline
\end{tabular}

According to the comparison, the evaluations provided by our scheme roughly agree with the conclusion from human expert, which means the method is feasible and the choice of threshold is practical in this situation. Furthermore, the accuracy is estimated by choosing 5 testers to performance 40 samples, and the consistency between the evaluation of our system and experts is about $82 \%$.

\section{Conclusion}

Motion evaluation automatically is commonly needed in many sports events, especially for those non-professional trainers. In this paper, we proposed an evaluation system based on Android platform to detect the movement of human body with build-in 3D accelerometer of the smart phone. The feasibility is proved in our experiment, and the flexibility and convenience are approved by professional person. The exploration of related extension will be considered in our future work.

\section{Acknowledgment}

This research is supported by the Youth Talents Project of Beijing (YETP1711).

\section{References}

[1] Harms H, Amft O, Winkler R, et al. Ethos: Miniature orientation sensor for wearable human motion analysis[C]. Sensors, 2010 IEEE. 2010: $1037-$ 1042.

[2] Strohrmann C, Harms H, Tröster G, et al. Out of the lab and into the woods: kinematic analysis in running using wearable sensors [C].Proceedings of the 13th international conference on Ubiquitous computing. ACM, 2011: 119-122.

[3] Wei Yu, Yan Hongli, Guo Junqi, Bie Rongfang. Patient's Motion Recognition Based on SOM-Decision Tree[C]. Proceedings of the $8^{\text {th }}$ International Conference on Wireless Algorithms, Systems, and Applications.2013: 252-264

[4] Taki T, Hasegawa J, Fukumura T. Development of motion analysis system for quantitative evaluation of teamwork in soccer games[C]. Proceedings of the International Conference on Image. IEEE, 1996, 3: 815-818.

[5] Perš J, Bon M, Kovačič S, et al. Observation and analysis of large-scale human motion[J]. Human Movement Science, 2002, 21(2): 295-311.

[6] Nesbit S M, Serrano M. Work and power analysis of the golf swing $[\mathrm{J}]$. Journal of Sports Science and Medicine, 2005, 4(4): 520-533.

[7] Ghasemzadeh H, Loseu V, Jafari R. Wearable coach for sport training: A quantitative model to evaluate wrist-rotation in golf[ $\mathrm{J}]$. Journal of Ambient Intelligence and Smart Environments, 2009, 1(2): 173-184. 
[8] Yang J. Toward physical activity diary: motion recognition using simple acceleration features with mobile phones[C]. Proceedings of the 1st international workshop on Interactive multimedia for consumer electronics. ACM, 2009: 1-10.

[9] Ståhl O, Gambäck B, Turunen M, et al. A mobile health and fitness companion demonstrator[C]. Proceedings of the 12th Conference of the European Chapter of the Association for Computational Linguistics: Demonstrations Session. Association for Computational Linguistics, 2009: 65-68. 